\title{
Dissociation of Toluene Cation: a New Potential Energy Surface
}

\author{
Joong Chul Choe \\ Department of Chemistry, University of Suwon, PO Box 77, Suwon 440-600, Korea \\ Tel.: +82-31-220-2150; Fax.: +82-31-222-9385 \\ E-mail: jcchoe@suwon.ac.kr
}

\section{Supporting Information}

Table S1. Cartesian coordinates of relevant species optimized at the B3LYP/6$311++\mathrm{G}^{* *}$ level.

Table S2. Vibrational frequencies $\left(\mathrm{cm}^{-1}\right)$ for $\mathrm{C}_{7} \mathrm{H}_{8}{ }^{+\bullet}$ ions calculated at the B3LYP/6$31 \mathrm{G}^{*}$ level. $i$ denotes the imaginary frequency.

Table S3. Vibrational frequencies $\left(\mathrm{cm}^{-1}\right)$ for $\mathrm{C}_{7} \mathrm{D}_{8}{ }^{+\bullet}$ ions calculated at the B3LYP/6$31 \mathrm{G}^{*}$ level. $i$ denotes the imaginary frequency.

Table S4. Reaction path degeneracies $(\sigma)$ assumed in RRKM calculations.

Table S5. RRKM rate expressions for $k_{1}-k_{9}$. 
Table S1. Cartesian coordinates of relevant species optimized at the B3LYP/6$311++\mathrm{G}^{* *}$ level.
Toluene $^{+\bullet}(\mathbf{1})$
C, $-1.2402529119,0.0087977949,-0.1862686761$
C, $-1.2385192411,-0.0092030429,1.1817368494$
C, $0.0001499703,-0.0189157429,1.8810970901$
C, $1.2387076531,-0.0092030429,1.1815393527$
C, $1.2402231956,0.0087977949,-0.1864664318$
$\mathrm{H},-2.1714623544,0.0171958737,-0.7406587732$
$\mathrm{H},-2.1669114887,-0.015365404,1.7395387456$
$\mathrm{H}, 0.0002364379,-0.0355288878,2.9656714669$
H, 2.1671888303, -0.015365404, 1.7391932099
$\mathrm{H}, 2.1713442289,0.0171958737,-0.7410050031$
C, $-0.0000730067,0.0217565124,-0.9157320655$
C, $-0.0001906224,0.0180488389,-2.391001083$
$\mathrm{H},-0.8994565909,0.4730289093,-2.8104806008$
$\mathrm{H},-0.0002177339,-1.0346645444,-2.7310652768$
H, $0.8990084489,0.4730289093,-2.8106239832$
$\mathrm{C}_{6} \mathrm{H}_{6} \mathrm{CH}_{2}^{+\bullet}$ (2)
C, $-1.25431853,0.0180954374,-1.1430494698$
C, $-1.2544221221,-0.0294426155,0.2334508746$
C, $0.0084157094,-0.1672776802,1.0020353285$
C, $1.2546530173,-0.1282491535,0.1958551327$
C, 1.2171090916, - $0.0792721781,-1.180073073$
C, $-0.0275596025,0.0025881962,-1.8461015118$
H, -2.1869257204, 0.0870906202, -1.6903340734
H, -2.1856003799, -0.0124972726, 0.7920514306
$\mathrm{H}, 2.2005686222,-0.1851870157,0.7263430487$
H, 2.1354576563, -0.0831875435, -1.7550993245
H, -0.0417950474, 0.0535391766, -2.9305786948
C, 0.0526511644, 0.4327227018, 2.3759420393
H, -0.868712442, 0.6047355652, 2.9191309142
H, 1.0003293509, 0.5293367039, 2.8918157673
H, -0.0324944077, -1.2888184829, 1.2183150095 


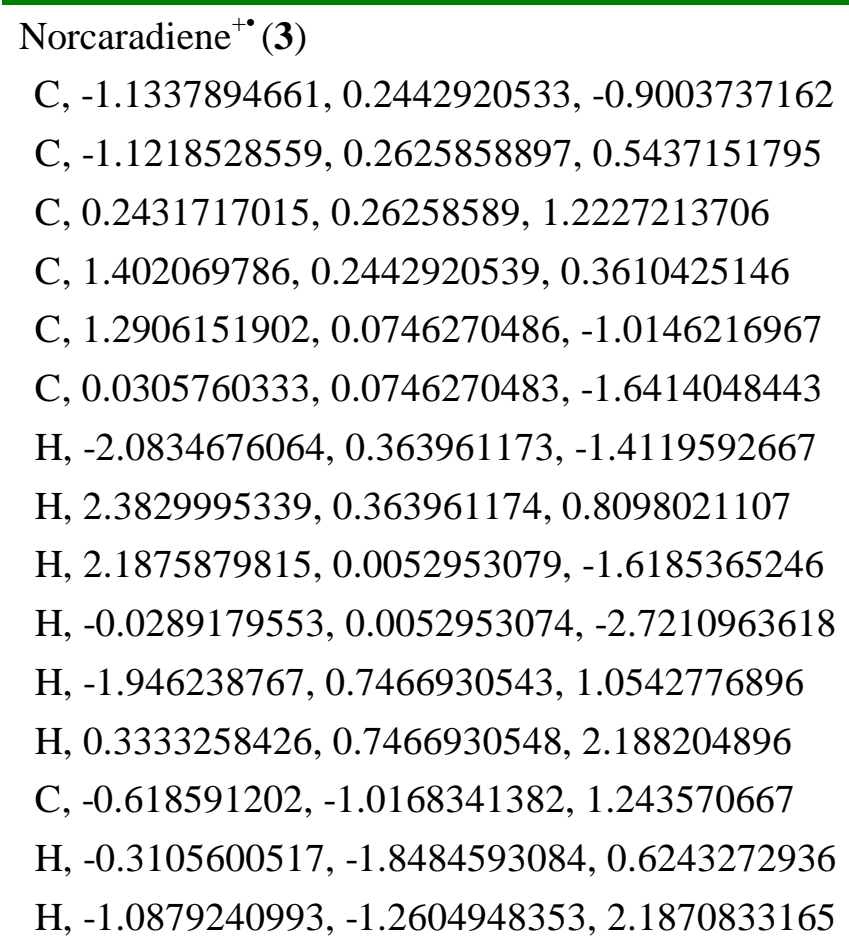

Cycloheptatriene $^{+\bullet}$ (4)

C, -1.365717226, 0., -0.937332202

C, $-1.516755674,0 ., 0.545254859$

C, -0.5715190796, 0., 1.5454942852

C, $0.8457427311,0 ., 1.4276219825$

C, $1.6362450074,0 ., 0.2758397503$

C, 1.2366222226, 0., -1.0890142894

C, $-0.036600605,0 .,-1.6113693033$

H, -2.5561113305, 0., 0.8694330675

H, -0.9516967676, 0., 2.5630341655

H, 1.3884290007, 0., 2.3692380908

H, 2.7100185849, 0., 0.443647935

H, 2.0494030688, 0., -1.809645803

H, - $-0.1078305218,0 .,-2.6977753004$

$\mathrm{H},-1.9501581468,0.8549124511,-1.3384513245$

$\mathrm{H},-1.9501581468,-0.8549124511,-1.3384513245$

$o$-Isotoluene ${ }^{+\bullet}(\mathbf{5})$

C, $-1.1963458504,0 .,-1.1922753104$ 

C, $-1.267713171,0 ., 0.2910321801$
C, $0.0575467774,0 ., 1.0157882539$
C, $1.255526017,0 ., 0.2483843177$
C, 1.2282411446, 0., - 1.1487796772
C, $0.0033468153,0 .,-1.8696828298$
H, -2.1344684222, 0., -1.7395430832
$\mathrm{H}, 2.2103724269,0 ., 0.7643849351$
H, 2.1641459718, 0., - 1.6975819667
$\mathrm{H}, 0.0226414844,0 .,-2.9532671116$
C, $0.1187418762,0 ., 2.3991186809$
H, -0.776199557, 0., 3.0135678092
$\mathrm{H}, 1.0718893356,0 ., 2.9182515947$
$\mathrm{H},-1.8772214472,-0.8636783799,0.6163370651$
H, -1.8772214472, 0.8636783799, 0.6163370651

$m$-Isotoluene ${ }^{+\bullet}(\mathbf{5 m})$

C, $-1.2213158144,0 .,-1.1526267037$

C, $-1.1985571975,0 ., 0.3205825924$

C, $0.0076066664,0 ., 1.0488104022$

C, $1.2220205109,0 ., 0.275611268$

C, $1.2585080018,0 .,-1.1296604224$

C, $0.0773885654,0 .,-1.8465828893$

H, 2.164699437, 0., 0.8170876846

H, 2.214940562, 0., -1.6386261887

H, $0.082979423,0 .,-2.9323384995$

C, $0.0547569108,0 ., 2.4663534218$

H, -0.853807796, 0., 3.0567806246

$\mathrm{H}, 0.9994826586,0 ., 2.9971232928$

H, $-2.1498226598,0 ., 0.844314562$

$\mathrm{H},-1.8304587427,0.853838565,-1.5196337447$

H, - $1.8304587427,-0.853838565,-1.5196337447$

$p$-Isotoluene ${ }^{+*}(\mathbf{5 p})$

C, $-1.2486863927,0 .,-1.0726952255$

C, $-1.2484553144,0 ., 0.296043716$

C, $-0.0076488422,0 ., 1.0294282003$ 

C, 1.243918397, 0., 0.3145625144
C, 1.2644882948, 0., -1.0540218719
C, 0.0137546027, 0., -1.8511789661
H, 2.1691755305, 0., 0.8804310268
H, 2.2088522319, 0., - 1.5895508061
C, $-0.0181051844,0 ., 2.4367070011$
H, -0.9496414243, 0., 2.9933366048
$\mathrm{H}, 0.9050569397,0 ., 3.0071173572$
H, -2.1820188426, 0., 0.8481008466
H, -2.1849883444, 0., -1.6221978555
H, $0.0189852727,0.857489347,-2.5551546917$
H, 0.0189852727, - $0.857489347,-2.5551546917$

Benzylium $^{+}$
C, $-1.2442533162,0 .,-0.2534302086$
C, $-1.2331670447,0 ., 1.1193188986$
C, $0.0001738137,0 ., 1.7991691547$
C, $1.2333840737,0 ., 1.1190809333$
C, $1.2442044576,0 .,-0.2536707831$
H, -2.1776369152, 0., -0.8049987544
H, -2.1585687702, 0., 1.6813044108
H, $0.0002783983,0 ., 2.88432369$
H, 2.1588945553, 0., 1.6808870349
H, 2.1774812829, 0., -0.8054199924
C, - $0.0000951722,0 .,-0.9816975197$
C, - $0.0002274491,0 .,-2.3493962895$
H, - $0.9262439752,0 .,-2.9160809792$
H, $0.9256792478,0 .,-2.9162605236$

Tropylium $^{+}$

C, -1.4494046336, 0., -0.6979964839

C, $-1.4494046336,0 ., 0.6979964839$

C, $-0.357973382,0 ., 1.5683838629$

C, $1.0030191275,0 ., 1.2577462039$

C, 1.6087177762, 0., 0 .

C, $1.0030191275,0 .,-1.2577462039$ 
C, $-0.357973382,0 .,-1.5683838629$

H, -2.4274733618, 0., 1.1690095589

H, -0.5995364089, 0., 2.6267406353

$\mathrm{H}, 1.6798636882,0 ., 2.1064824377$

H, 2.6942921651, 0., 0 .

H, 1.6798636882, 0., -2.1064824377

H, -0.5995364089, 0., -2.6267406353

H, -2.4274733618, 0., -1.1690095589

\section{TS12}

C, - $1.2442086954,0.0046810224,-1.148058657$

C, $-1.2463074512,0.0371286011,0.222215304$

C, $0.0122624658,-0.0186422729,0.9645338135$

C, $1.2498503655,-0.0805798462,0.1882057106$

C, 1.207459259, -0.1109294676, -1.1814620859

C, $-0.0281668739,-0.0615066381,-1.8544506159$

H, -2.1807718297, 0.0395128734, -1.6909170791

H, -2.1760560856, 0.084371768, 0.7780971275

H, 2.1946856634, - $0.1217342809,0.7185467461$

H, 2.1280403606, -0.1636728344, -1.7496236841

H, $-0.0440402183,-0.085019554,-2.9381067329$

C, $0.04808835,0.3279238794,2.3945214186$

H, -0.8771782454, 0.4050188251, 2.9513479422

H, 0.9912974403, 0.3169092632, 2.9258903778

H, - $0.0298416041,-1.063837729,1.4917359747$

\section{TS23}

C, $-1.0418621313,0.2112060428,-0.7249337609$

C, $-0.7771239754,-0.2685689671,0.6466671763$

C, $0.6289663109,-0.1473768506,1.1026623022$

C, $1.5972528582,0.4039017817,0.3071242637$

C, 1.2528185198, 0.8510810567, - 0.9838233341

C, $-0.0574481193,0.7672606215,-1.4964932512$

$\mathrm{H},-2.0580184425,0.1231339137,-1.0955985967$

H, 2.6202896133, 0.4893189724, 0.6522681874

H, 2.0298746676, 1.2790668721, -1.61025291 
H, $-0.2689393551,1.1279618062,-2.4954413063$

H, -1.3153886127, 0.4775910028, 1.2840481823

$\mathrm{H}, 0.862880762,-0.5027964939,2.1007151152$

C, -1.3952079823, -1.613521164, 0.9741373595

$\mathrm{H},-1.3897943662,-2.3974759238,0.2300710808$

H, -1.7252771495, -1.820695275, 1.9821457145

\section{TS25}

C, $1.1898954665,-1.2048360742,-0.0397888811$

C, $-0.2052357093,-1.2428874483,-0.014663728$

C, $-0.9914789332,0.0014225609,0.0643672586$

C, $-0.2346588919,1.2475067519,0.0411434184$

C, $1.1382443938,1.2396116155,-0.0034138332$

C, $1.8528061707,0.0192100552,-0.0533813953$

H, 1.7441940524, -2.1365730758, - 0.0695711758

H, $-0.747071948,-2.1839983653,0.0083374831$

H, -0.7865822985, 2.1817929918, 0.0639065819

H, 1.6820646396, 2.1783917026, -0.0121034284

H, 2.9375755776, 0.0393998972, - 0.0896808637

C, $-2.4337120476,-0.0024591009,-0.1178217096$

$\mathrm{H},-2.9872215687,-0.9332613748,-0.1473165198$

$\mathrm{H},-2.9745317912,0.933553505,-0.1777740243$

H, $-0.7635893576,-0.4247154414,1.1655551683$

\section{TS34}

C, $-1.2212366835,0.6371966892,-0.6351442174$

C, $-1.3829963411,-0.063030114,0.5216209501$

C, $0.5214650147,-0.2905729079,1.2667842224$

C, $1.5039107751,0.1293218547,0.3124185996$

C, $1.2331423479,0.3526616961,-1.0303666766$

C, $-0.0643410753,0.5763914241,-1.4891313067$

H, -2.014349575, 1.3160199342, -0.9358836744

$\mathrm{H}, 2.5156551185,0.2694053874,0.687681704$

H, 2.0608892044, 0.4998038451, -1.7138300346

H, -0.1990591009, 0.8570149457, -2.5291974332

H, -2.1981286181, 0.1776889862, 1.1946272399 
H, 0.5718930584, 0.093572869, 2.2830135599

C, $-0.5105210858,-1.2593464529,0.9176817681$

$\mathrm{H},-0.2731340445,-1.9073644169,0.0759679709$

H, - $0.9403037549,-1.8018746867,1.75444063$

\section{TS55m}

C, $-1.2651069538,0.0851678258,-1.076014666$

C, $-1.1737313168,0.1343147401,0.3715075858$

C, $0.1031490848,0.022417491,1.027683974$

C, $1.2540783472,-0.0869743748,0.1824302789$

C, $1.1604833083,-0.1033477594,-1.2144393802$

C, $-0.075114282,-0.0162743837,-1.8607375142$

H, -2.218016777, 0.3102732357, -1.5460354509

H, 2.2304648057, $-0.1545831101,0.6503558562$

H, 2.0671688874, - $0.1777996461,-1.8038258558$

H, $-0.1494442216,-0.0212592051,-2.9407985224$

C, 0.1899524423, 0.0614264782, 2.428142376

H, -0.6942479973, 0.1436424248, 3.0501959399

$\mathrm{H}, 1.1504172594,0.0241548501,2.9290517593$

$\mathrm{H},-1.4675813203,-0.9964483457,-0.4368971173$

H, -2.0810244159, 0.2916396933, 0.9465174654

\section{TS5m5p}

C, $-1.2002952027,-0.0371186069,-1.135317752$

C, $-1.2275350239,-0.0402933119,0.2829000581$

C, $-0.0225048355,-0.0102825325,1.0330791069$

C, 1.2263530748, 0.0071329418, 0.3046103315

C, $1.2809451598,0.0086501674,-1.0743986115$

C, 0.0802020556, - $0.0319245837,-1.835163754$

$\mathrm{H}, 2.1503714066,0.0193505984,0.8735864736$

H, 2.2326847385, 0.0180323954, -1.5913671768

H, 0.0918386698, - $0.1164139991,-2.916191282$

C, $-0.0326156957,-0.0106803342,2.4444943569$

$\mathrm{H},-0.9634102132,-0.0276885268,2.9998877354$

$\mathrm{H}, 0.890135681,0.0025208416,3.0130996122$

H, -2.1845116324, $-0.0770566214,0.7904636132$ 
H, - $0.7347441872,1.075001755,-1.5873868745$

H, -2.1096616577, -0.2066488829, - 1.703314516

\section{TS4Tr}

C, $-1.4128845545,-0.0232816878,-0.6867999305$

C, $-1.4134883044,-0.0106920932,0.7201426355$

C, $-0.3279009188,-0.0031104264,1.5871885754$

C, $1.0390680293,0.0040533852,1.2787447763$

C, $1.6462386138,0.0635083789,0.0277116213$

C, $1.0416476923,0.1309826331,-1.2347079923$

C, $-0.3104044391,0.0969972441,-1.5526810488$

H, -2.3937105465, -0.0229797563, 1.186632527

H, -0.5701060646, -0.0054189509, 2.6454254145

H, 1.7123948032, - $0.0193800815,2.1299339329$

H, 2.7316660398, 0.08031987, 0.0298248593

$\mathrm{H}, 1.721521236,0.2192118974,-2.076430463$

$\mathrm{H},-0.5513130501,0.1568721428,-2.6095522598$

H, -2.3887571204, 0.0433899917, -1.157282535

H, -1.8353520082, -2.002759717, -0.9861432964

\section{TS5Bz}

C, $-1.2114042553,-0.0479573586,-1.1277856566$

C, $-1.2118564765,-0.0500249975,0.2603522253$

C, $0.0479121017,-0.0572871226,0.9835652456$

C, 1.2843717582, 0.0064153801, 0.2335093623

C, $1.2572214943,0.0318658282,-1.1456119159$

C, $0.0137620034,-0.0069924739,-1.82314825$

H, -2.1447613095, -0.0750130612, -1.6767932635

H, 2.2257616206, 0.0233959211, 0.7728437343

H, 2.1779005221, 0.0720665593, -1.7148115387

H, 0.0050207283, $-0.0075812025,-2.9085007911$

C, $0.0659004631,-0.154261541,2.3533910763$

H, $-0.850743111,-0.2121603538,2.934343428$

H, $0.9988508607,-0.1807300571,2.9100618664$

H, -2.1394393676, -0.1371458269, 0.8167494582

H, -1.7480324774, 2.1866217323, 0.4604745844 


\section{TS5pBz}

C, -1.2416237354, 0.045542526, - 1.0604512117

C, -1.2416177529, 0.0443344798, 0.3108062301

C, $0.0065284329,0.0427941629,1.0325289729$

C, $1.2453482173,0.0480128832,0.2949292863$

C, $1.2278429938,0.0491950468,-1.0762164393$

C, $-0.011256925,0.0425267734,-1.7534330848$

$\mathrm{H}, 2.1836481886,0.0567342866,0.8391611764$

H, 2.1509407686, 0.0592566268, -1.6432485053

C, $0.0152681114,0.039891155,2.4008404665$

H, -0.905421504, 0.0368929472, 2.9761332569

$\mathrm{H}, 0.9432326167,0.039627241,2.9643313352$

H, -2.1729144159, 0.0502906138, 0.8669737403

H, -2.1719116174, 0.0528628139, -1.6156511495

H, $-0.0182542019,0.0955890456,-2.8371936279$

H, $-0.0122558874,-2.2650357376,-2.4445315459$ 
Table S2. Vibrational frequencies $\left(\mathrm{cm}^{-1}\right)$ for $\mathrm{C}_{7} \mathrm{H}_{8}{ }^{+\bullet}$ ions calculated at the B3LYP/6$31 \mathrm{G}^{*}$ level. $i$ denotes the imaginary frequency.

\begin{tabular}{|c|c|}
\hline 1 & $\begin{array}{l}37^{\mathrm{a}} 152346354388496513577746780801944990990998 \\
101010121028109111721224126213111386140114131454 \\
148115041547168829673097315532213223322732383242\end{array}$ \\
\hline 2 & $\begin{array}{l}148192302374398511544595624733752784857910949 \\
97110231031103510571131118012111232133214001466 \\
149514971579164526763195321532173221323532383312\end{array}$ \\
\hline 3 & $\begin{array}{l}2342723754555395996647007638058648959359621019 \\
102510411052106611081125119511981223137313871464 \\
148515221566157031833196319832133216322832373287\end{array}$ \\
\hline 4 & $\begin{array}{l}98221366413425516630798807810878890942947966 \\
101410451047114112281258128313241395142814641496 \\
153615831607166529392945318631873195320332093214\end{array}$ \\
\hline 5 & $\begin{array}{l}119248361418474502586593729785840919933985986 \\
99510141040107111971199121413261355139314331458 \\
152615481585160829963009318232133217322632373279\end{array}$ \\
\hline $5 \mathrm{~m}$ & $\begin{array}{l}176216319363453488506595672771797799896934962 \\
98110151054107711431153121412651324135913971439 \\
149015151570160129612962319432033219322232373296\end{array}$ \\
\hline $5 p$ & $\begin{array}{l}151297334363485517552602776788814880900961974 \\
9879931035104011681177123013251357136214141485 \\
149915121540169129662967318532153215322732293288\end{array}$ \\
\hline TS12 & $\begin{array}{l}859 i 168329373447450523603638758789798813905972 \\
9779771019102610351134118912051241134513921466 \\
147914971573163624843190321532163225323432393312\end{array}$ \\
\hline TS23 & $\begin{array}{l}126 i 133256358382466525590613754801807924974983 \\
102310331056106710921122114112101226131714021460 \\
149014951581164427973201321232183221323632383322\end{array}$ \\
\hline
\end{tabular}


TS25

TS34

TS55m

TS5m5p

TS4Tr

TS5Bz

TS5pBz

$\operatorname{TS1Bz}(1.6 \AA)$
$773 i 208295337377466528611637667780810863932950$ 97710131032103910521129121212131267132913751486 149115041572164022553199322132243229323632413311 $353 i 240289388433540636707782836866891908953988$ 99210061051111211611189121712311246137313921427 145815201542161331243180319331943205320932223228 $859 i 185353376431512518614654742821844851931971$ 98310211031106711121177120712391312135813951454 151015291555157622013192320732183222323132503293 $892 i 186351359472482524610653740825836862905974$ 98210081028108111121166121512291298136213961473 150415261546161921363192321432163232323532433294

$675 i 187226292331439441564575693873883896900913$ 100510161056106010751079124912611315131914471522 152315641567162716423193319532023203321132123217

$409 i 154195238354362427534610634661796823856988$ 99510041012102910511099113912101218136013961431 148115141582160716683179321732213224323932423275

$236 i 144147209347360436534610629658801824851991$ 99810031013102110431097114012081222136313961433 149115131581160616753179321732203223323932413275

$1060 i 198349365428522559574629781799817938980999$ 100010081010102811251174118911901225131413481408 143814941521155016763158322032213226323832413250

TS1Bz (2.0 Å) ～1075i 190348354425443508569602651791801826856991 99210041011102510401080114012041222135713621419 148015091559157216673175321732193223323832413274

TS1Bz (2.6 ̊̊) ～256i 148162213351364427534611648654801826852992 100110041013102910511089114212101221136413901430 148815151587160016713181321732203222323932423277

${ }^{a}$ This torsional mode was replaced by the internal rotation (rotational constant of 5.5 $\mathrm{cm}^{-1}$ ) of the methyl group in RRKM calculations. 
Table S3. Vibrational frequencies $\left(\mathrm{cm}^{-1}\right)$ for $\mathrm{C}_{7} \mathrm{D}_{8}{ }^{+}$ions calculated at the B3LYP/631G* level. $i$ denotes the imaginary frequency.

\begin{tabular}{|c|c|}
\hline 1 & $\begin{array}{l}26^{\mathrm{a}} 135298309330456482485581622711750789816838 \\
8458498588868899581026103110751080121512541340 \\
13441466164821502268234123792379238524002405\end{array}$ \\
\hline 2 & $\begin{array}{l}133137260320347405487492524580626659702704750 \\
828845850859877878887947106510731188128513171458 \\
1501159819552313237423742382239724022473\end{array}$ \\
\hline 3 & $\begin{array}{l}205238316390504565569585653653691694752804812 \\
82784986787587788589895396210741105127212801409 \\
1497153723072360236123712376238823992455\end{array}$ \\
\hline 4 & $\begin{array}{l}81194306396411436465614641738768780795820822824 \\
869877882925932955964101911101247126814071548 \\
1550163421472165234823512357236723722377\end{array}$ \\
\hline 5 & $\begin{array}{l}101209307353377440478561568656708751777789798 \\
838856873884887915918102410761080123912861428 \\
14591545155521832221231523722375238823992446\end{array}$ \\
\hline $5 \mathrm{~m}$ & $\begin{array}{l}155167274298338418483526567596637706743761790 \\
82083386887788390193796910621072123312911349 \\
14411504154821622179231823662375238124012460\end{array}$ \\
\hline $5 p$ & $\begin{array}{l}128245276310360446490578595652711738763775796 \\
80080685688188390591799610731082124212871422 \\
14471473165021642185231523712374239123962454\end{array}$ \\
\hline TS12 & $\begin{array}{l}654 i 150283304340388490495572594596675691717765 \\
800827849855862873878948106510731193130513181430 \\
1492158818182308237423742384239624022474\end{array}$ \\
\hline TS23 & $\begin{array}{l}97 i 109217304327392473503563608623694707743829 \\
840851863876878881901947105710711146126013031462 \\
1500159820452315237323752381239824032481\end{array}$ \\
\hline
\end{tabular}


TS25

TS34

TS55m

TS5m5p

TS4Tr

TS5Bz

TS5pBz
$594 i 184211288325396493511527585648676692736764$

79582785485786687688994910681074122812611362

14041514158216652318237823792388239724032472

$325 i 206254338395447535554620678721747784799811$ 826844857862894911931987100010851120123713211344 1460156922722344235423592367238223872390

$664 i 165301310367381491519588604668681736775777$ 80184585586086688391895310701079126213101361 14121489151216282318236923762381239024072457

$691 i 165290306355408496517581599668672747774781$ 80184084685087287892195110681078124313101345 14221480156515902318237423752388239324032458

501i 150191219263423423475479528683694825832833 8378398448498848949249299679691117129613051536 1548155815662356235723632364237323742379

$299 i 121160175299308375458508519586660669746790$ 79882285586387687788489696110771091128613441440 1510153216222321237723792384239924042443

$170 i 107112175294308382453508515586666667747792$ 80482685786086487588389796210771091128313501450 1510153016312320237623782383240024052444

TS1Bz (2.0 ̊̊) $\quad 798 i 167275301345353410503518566584664674747789$ 79582284785786187387688596410781085127513471431 1459149116242311237623772383239924042443

${ }^{\mathrm{a}}$ This torsional mode was replaced by the internal rotation (rotational constant of 2.8 $\mathrm{cm}^{-1}$ ) of the methyl group in RRKM calculations. 
Table S4. Reaction path degeneracies $(\sigma)$ assumed in RRKM calculations.

\begin{tabular}{|c|c|c|}
\hline Reactant & $\mathrm{TS}$ & $\sigma$ \\
\hline 1 & TS12 & $3^{\mathrm{a}}$ \\
\hline 1 & TS1Bz & $3^{\mathrm{a}}$ \\
\hline 2 & TS12 & 1 \\
\hline 2 & TS23 & 2 \\
\hline 2 & TS25 & 2 \\
\hline 3 & TS23 & 2 \\
\hline 3 & TS34 & 1 \\
\hline 4 & TS34 & 2 \\
\hline 4 & TS4Tr & 2 \\
\hline 5 & TS25 & 2 \\
\hline 5 & TS55m & 2 \\
\hline 5 & TS5Bz & 2 \\
\hline $5 \mathrm{~m}$ & TS55m & 2 \\
\hline $5 \mathrm{~m}$ & TS5m5p & 2 \\
\hline $5 p$ & TS5m5p & 4 \\
\hline $5 p$ & TS5pBz & 2 \\
\hline
\end{tabular}

${ }^{a}$ The symmetry number of the internal rotation of $\mathrm{CH}_{3}$. 
Table S5. RRKM rate expressions for $k_{1}-k_{9}{ }^{\text {a }}$

\begin{tabular}{|c|c|}
\hline$k$ & Expression \\
\hline \multirow{2}{*}{$k_{1}$} & $\underline{3 N_{\mathrm{TS} 12}^{\ddagger}} \quad 2 N_{\mathrm{TS} 23}^{\neq}$ \\
\hline & $\overline{h \rho_{1}} \overline{N_{\mathrm{TS} 12}^{\neq}+2 N_{\mathrm{TS} 23}^{\neq}+2 N_{\mathrm{TS} 25}^{\neq}}$ \\
\hline \multirow{2}{*}{$k_{2}$} & $4 N_{\mathrm{TS23}}^{\neq}-N_{\mathrm{TS} 12}^{\neq}$ \\
\hline & $\overline{h\left(2 \rho_{3}+\rho_{4}\right)} \overline{N_{\mathrm{TS} 12}^{\neq}+2 N_{\mathrm{TS} 23}^{\neq}+2 N_{\mathrm{TS} 25}^{\neq}}$ \\
\hline \multirow{2}{*}{$k_{3}$} & $2 N_{\mathrm{TS} 4 \mathrm{Tr}}^{\neq}$ \\
\hline & $\overline{h\left(2 \rho_{3}+\rho_{4}\right)}$ \\
\hline \multirow{2}{*}{$k_{4}$} & $\underline{3 N_{\text {TS1Bz }}^{\ddagger}}$ \\
\hline & $h \rho_{1}$ \\
\hline \multirow{2}{*}{$k_{5}$} & $3 N_{\mathrm{TS} 12}^{\neq} \quad 2 N_{\mathrm{TS} 25}^{\neq}$ \\
\hline & $h \rho_{1} \quad \overline{N_{\mathrm{TS} 12}^{\neq}+2 N_{\mathrm{TS} 23}^{\neq}+2 N_{\mathrm{TS} 25}^{\neq}}$ \\
\hline \multirow{2}{*}{$k_{6}$} & $4 N_{\mathrm{TS} 25}^{\neq}$ \\
\hline & $\overline{h\left(2 \rho_{5}+2 \rho_{5 \mathrm{~m}}+\rho_{5 \mathbf{p}}\right)} \overline{N_{\mathrm{TS} 12}^{\neq}+2 N_{\mathrm{TS} 23}^{\neq}+2 N_{\mathrm{TS} 25}^{\neq}}$ \\
\hline \multirow{2}{*}{$k_{7}$} & $4 N_{\mathrm{TS} 5 \mathrm{Bz}}^{\neq}+2 N_{\mathrm{TS} 5 \mathrm{pBz}}^{\neq}$ \\
\hline & $\overline{h\left(2 \rho_{5}+2 \rho_{5 \mathrm{~m}}+\rho_{5 \mathrm{p}}\right)}$ \\
\hline \multirow{2}{*}{$k_{8}$} & $4 N_{\mathrm{TS} 25}^{\neq}$ \\
\hline & $\overline{h\left(2 \rho_{5}+2 \rho_{5 \mathrm{~m}}+\rho_{5 \mathrm{p}}\right)} \overline{N_{\mathrm{TS} 12}^{\neq}+2 N_{\mathrm{TS} 23}^{\neq}+2 N_{\mathrm{TS} 25}^{\ddagger}}$ \\
\hline \multirow{2}{*}{$k_{9}$} & $4 N_{\mathrm{TS23}}^{\neq} \quad 2 N_{\mathrm{TS} 25}^{\neq}$ \\
\hline & $\overline{h\left(2 \rho_{3}+\rho_{4}\right)} \overline{N_{\mathrm{TS} 12}^{\neq}+2 N_{\mathrm{TS} 23}^{\neq}+2 N_{\mathrm{TS} 25}^{\neq}}$ \\
\hline
\end{tabular}

a The symmetry number (3) of the internal rotation of $\mathrm{CH}_{3}$ was not included in the count of density states of $\mathbf{1}$. Instead, it was used as the reaction path degeneracy. 